19 Revue d'histoire du XIXe siècle

Société d'histoire de la révolution de 1848 et des

révolutions du XIXe siècle

56 | 2018

Un autre $\mathrm{XIX}^{\mathrm{e}}$ siècle : I'Inde sous domination coloniale

\title{
Les résistances des marins indiens
}

Justine Cousin

URL : http://journals.openedition.org/rh19/5555

DOI : $10.4000 /$ rh19.5555

ISSN : $1777-5329$

Éditeur

La Société de 1848

Édition imprimée

Date de publication : 15 octobre 2018

Pagination : 140-142

ISSN : 1265-1354

Référence électronique

Justine Cousin, «Les résistances des marins indiens », Revue d'histoire du XIXe siècle [En ligne], 56 |

2018, mis en ligne le 15 octobre 2020, consulté le 05 janvier 2021. URL : http://

journals.openedition.org/rh19/5555; DOI : https://doi.org/10.4000/rh19.5555 


\section{JUSTINE COUSIN \\ Les résistances des marins indiens}

Au début du XXe siècle, les marins travaillant sur les navires à vapeur britanniques sont généralement caractérisés par leur militantisme et leur résistance vis-à-vis de leurs employeurs et des autorités politiques, comme le souligne l'historien indien Gopalan Balachandran ${ }^{39}$. Au sein de cette population, les marins coloniaux sont engagés de manière croissante à bord de ces navires à partir du milieu du XIX ${ }^{e}$ siècle. Les supérieurs blancs exercent alors une discipline de fer sur cette main-d'œuvre à moindre coût, obéissante, qui travaille dans des conditions difficiles telles que dans la salle des machines extrêmement bruyante et dangereuse avec une chaleur étouffante, dans le service hôtelier et ses horaires à rallonge ou bien encore sur le pont sous la chaleur tropicale et le danger des éléments extérieurs. Les Indiens - aussi appelés lascars ${ }^{40}-$ forment le plus gros contingent de ces marins de couleur, atteignant, avec plus de 50000 hommes en 1938, plus du quart de la totalité des marins engagés sur des navires britanniques $^{41}$.

Les deux tendances historiographiques ayant traité récemment cette question insistent sur «la capacité [des marins indiens] à remettre en cause et à résister à des pratiques visant à les maintenir dans une position subordonnée» comme le souligne Fidler. Cette capacité peut résulter d'un raisonnement calculé ou d'un déchaînement de violence aveugle selon les historiens, l'essentiel étant que ces marins indiens ne subissent pas impuissants les conditions de travail et de vie à bord du navire car ils ont le pouvoir - l'agency - «de prendre le contrôle d'une partie de leurs vies ${ }^{42}$ de manière violente ou non, collectivement ou individuellement, que ce soit par la contrebande, le refus de travailler, l'agression d'un supérieur ou bien encore la désertion. Les syndicats font également partie de la prise de pouvoir de ces marins longtemps perçus comme impuissants; ils ont notamment été étudiés par Broeze et Balachandran, qui détaillent leurs débuts et leurs évolutions progressives en dépit de nombreux obstacles politiques et syndicaux en Grande-Bretagne et en Inde ${ }^{43}$.

Les historiens ont longtemps débattu de l'ampleur des résistances de ces marins indiens face à cette discipline draconienne. Dans la lignée des Subaltern Studies, les historiens de l'Asie du Sud ont concentré leurs recherches sur

39. Gopalan Balachandran, Globalizing Labour? Indian Seafarers and World Shipping, c. 18701945, Oxford, Oxford University Press, 2012, p. 264.

40. Le «lascar» était un artilleur indien au XVIII e siècle selon une racine perse et urdu ou un travailleur des mers selon une autre étymologie perse et tamoul.

41. British Parliamentary Papers, Annual statements of trade and navigation, 1886-1938.

42. Ceri-Anne Fidler, Lascars, c.1850-1950: The Lives and Identities of Indian Seafarers in Imperial Britain and India, thèse d'histoire sous la direction de Padma Anagol, Cardiff University, 2011, p. 58.

43. Cf. Frank Broeze, "Militancy and Pragmatism: an International Perspective on Maritime Labour, 1870-1914", International Review of Social History, volume 36, n² 2, 1991, p. 165-200, et Gopalan Balachandran, Globalizing Labour? ..., op. cit., chapitre 6. 
les rébellions telles que les révoltes paysannes, considérant la violence comme un attribut éminemment subalterne. Dans les années 1980, l'historien Ranajit Guha avance ainsi que le comportement raisonné et légaliste des élites diffère du comportement violent des subalternes ${ }^{44}$. Dans un travail plus récent, Balachandran remet en question cet argument : selon lui les protestations des lascars n'étaient pas arbitraires ou violentes mais plutôt rationnelles; ces hommes respectaient le cadre légal quand ils contestaient une situation injuste que leur faisaient subir leurs supérieurs ${ }^{45}$, comme lorsqu'ils n'étaient pas ramenés en Inde à la fin de leur contrat d'engagement - tel que le stipulait pourtant ce dernier pour tous les marins indiens - mais transférés sur un autre navire, ou lorsqu'ils ne recevaient pas la quantité de nourriture légalement promise lors de l'engagement. De telles insubordinations individuelles ou collectives avaient peu de chances d'aboutir favorablement face aux supérieurs blancs et il s'agissait le plus souvent de contestations ponctuelles d'une minorité de marins. Balachandran limite ainsi la portée de ces révoltes dans la mesure où les marins de couleurs n'osaient que très rarement remettre en question les règles édictées par leurs supérieurs à bord; les relations entre ces marins et leurs officiers étaient donc «stables", avec relativement peu de contestations ${ }^{46}$.

Certains historiens analysent au contraire le navire non pas comme "une toile de fond [...] statique, mais [plutôt comme] un champ de bataille permanent ${ }^{47}$, dans lequel les relations quotidiennes n'étaient pas aussi fluides que l'avance Balachandran. Les marins indiens utilisaient ainsi la violence comme un levier pour défendre leur économie morale, autrement dit « un certain niveau de droits, d'usages au travail et d'attentes auxquels ils pensaient avoir droit " ${ }^{48}$. Dès lors ces marins ciblaient tout particulièrement les individus qui avaient "violé les notions de justice» auxquelles ils donnaient de la valeur ${ }^{49}$. Lorsque Chitra Joshi décrit les actions envisagées par les travailleurs contre leurs agresseurs sous la forme de "harcèlement, d'humiliation ou d'agression physique» - elle souligne la pensée rationnelle accompagnant ces projets. Puisqu'il s'agissait de "questionner ou de redéfinir les limites de l'autorité ${ }^{50}$, les marins contestataires «retournaient [en leur faveur] les deux grands signes de leur subordination au quotidien : les injures et la violence physique ${ }^{51}$. Les journaux de l'époque se font ainsi l'écho de tels faits à bord ou de procès en découlant, comme dans le

44. Ranajit Guha, "On Some Aspects of the Historiography of Colonial India", in Ranajit Guha, Gayatri Chakravorty Spivak (eds), Selected Subaltern Studies, Oxford, Oxford University Press, 1988, p. 42.

45. Gopalan Balachandran, "Cultures of Protest in Transnational Contexts: Indian Seamen Abroad, 1886-1945”, Transforming Cultures eJournal, volume 3, nº 2, 2008, p. 49, 59.

46. Gopalan Balachandran, Globalizing Labour?..., op. cit., p. 53.

47. Ravi Ahuja, "Capital at Sea, Shaitan Below Decks? A Note on Global Narratives, Narrow Spaces, and the Limits of Experience", History of the Present, volume 2, n 1, printemps 2012, p. 81.

48. Ceri-Anne Fidler, Lascars, c.1850-1950..., op. cit., p. 63.

49. Idem, p. 62.

50. Chitra Joshi, Lost Worlds: Indian Labour and Its Forgotten Histories, Delhi, Permanent Black, 2003, p. 168.

51. Dipesh Chakrabarty, Rethinking Working-Class History: Bengal, 1890-1940, Delhi, Oxford India, 1996, p. 182. 
cas d'un navire en 1893 où les lascars blessent gravement le second ingénieur à bord alors que ce dernier leur demandait un pourboire pour les autoriser à boire de l'eau. Lors du procès qui suit l'officier nie le pourboire et les lascars sont accusés d'insubordination et d'agression sur l'officier ${ }^{52}$.

Justine Cousin prépare une thèse sur les marins de couleur employés par les compagnies maritimes impériales britanniques (1860-1960) à Sorbonne Université

\section{ÉRIC MEYER \\ L'impact des plantations sur la paysannerie à Ceylan : débats et combats}

L'île de Ceylan (aujourd'hui Sri Lanka) a été colonisée par le RoyaumeUni à partir de 1796 après l'avoir été par les Portugais et les Hollandais. Les Britanniques développèrent à partir des années 1840 dans les régions montagneuses de l'ancien royaume de Kandy une agriculture de plantation fondée sur le travail servile de coolies (Tamouls immigrés d'Inde du Sud) et destinée à l'exportation de café, puis à partir du dernier tiers du XIX ${ }^{\mathrm{e}}$ siècle, de thé, d'hévéas, et de cocotiers, qui empiéta sur l'agriculture vivrière des paysans autochtones cingalais fondée sur le paddy irrigué et sur les cultures sur brûlis. Dès la fin du siècle, des controverses opposèrent les défenseurs de la paysannerie aux promoteurs du développement colonial. À partir des années 1930, dans un contexte de crise de l'économie d'exportation, le mouvement nationaliste fit sien l'argumentaire "paysanniste ${ }^{53}$, ce qui conduisit à des politiques visant à «repaysanniser» le pays, tandis que, porté par la vague des peasant studies, le débat sur l'impact des plantations sur l'économie et la société paysannes prenait de l'ampleur dans le monde académique. La production historiographique des années 1960 à 1980 a été dominée par cette problématique, qui a été balayée du champ de la recherche par les ethnic studies, apparues dans le contexte de la guerre qui a déchiré le pays de 1983 à 2009.

Le débat prit forme dans le dernier quart du XIX ${ }^{\mathrm{e}}$ siècle, dans un contexte de crise résultant de l'effondrement de la production de café due aux ravages d'une maladie fongique. Une nouvelle génération d'administrateurs coloniaux se donna pour mission de défendre la paysannerie face aux planteurs. Ils s'inspiraient de l'image idéalisée de la société paysanne et de ses institu-

52. British Library, India Office Records, L/E/7/304, "Letter from Board of Trade Offices, Tilbury to Marine Department, Board of Trade", 5 juin 1893.

53. Ce terme est emprunté à la version française de Gordon Wright, La révolution rurale en France, Paris, L'Épi, 1967; sous sa forme anglaise, peasantrism, il a été forgé pour décrire les mouvements agrariens d'Europe centrale dans les années 1930, et il a été employé dans le cas ceylanais par Mick Moore, The State and Peasant Politics in Sri Lanka, Cambridge University Press, 1985. 